PROCEEDINGS OF THE

AMERICAN MATHEMATICAL SOCIETY

Volume 126, Number 4, April 1998, Pages 1101-1107

S 0002-9939(98)04232-4

\title{
TIGHT COMPACTLY SUPPORTED WAVELET FRAMES OF ARBITRARILY HIGH SMOOTHNESS
}

\author{
KARLHEINZ GRÖCHENIG AND AMOS RON \\ (Communicated by Palle E. T. Jorgensen)
}

\begin{abstract}
Based on Ron and Shen's new method for constructing tight wavelet frames, we show that one can construct, for any dilation matrix, and in any spatial dimension, tight wavelet frames generated by compactly supported functions with arbitrarily high smoothness.
\end{abstract}

\section{INTRODUCTION}

In 1988, Ingrid Daubechies constructed univariate compactly supported orthonormal wavelet bases of arbitrarily high smoothness (cf. [D1], [D2]). Her construction has been widely received, and rightly so, as a milestone in the theory and applications of wavelets, that affirmed the great practical importance of multiresolution analysis. However, generalizations of these constructions to higher dimensions and general dilation matrices turned out to be difficult. Aside from the straightforward tensor product construction of Daubechies' wavelet (that is tied to dyadic dilation, and dictates large rectangular support of the wavelet, and an exponential growth in the number of mother wavelets as the spatial dimension increases), only a few, specific, constructions of multivariate orthonormal wavelet systems exist presently in the literature. Indeed, even for the simple dilation matrix

$$
\left(\begin{array}{cc}
1 & -1 \\
1 & 1
\end{array}\right),
$$

it is still unknown whether there exists a scaling function in $C^{1}\left(\mathbb{R}^{2}\right)$ whose integer shifts are orthonormal (cf. Cohen and Daubechies [CD] for results on this topic).

Tight frames generalize orthonormal systems. They preserve the unitary property of the relevant analysis and synthesis operators, while sacrificing the orthonormality and the linear independence of the system (in order to get more flexibility). Precisely, a system $X \subset L_{2}\left(\mathbb{R}^{d}\right)$ is a tight frame if the analysis operator associated with $X$ is unitary, i.e., if, for every $f \in L_{2}\left(\mathbb{R}^{d}\right)$,

$$
\|f\|_{2}^{2}=\sum_{x \in X}|\langle f, x\rangle|^{2} .
$$

Received by the editors September 23, 1996.

1991 Mathematics Subject Classification. Primary 42C15; Secondary 42C30.

Key words and phrases. Affine systems, frames, tight frames, multiresolution analysis, wavelets.

This work was supported by the National Science Foundation under Grants DMS-9224748 and DMS-9626319, and by the U.S. Army Research Office under Contract DAAH04-95-1-0089.

(C) 1998 American Mathematical Society 
This identity implies that

$$
\sum_{x \in X}\langle f, x\rangle x=f, \quad \text { all } f \in L_{2},
$$

hence entitles one to use the same system $X$ for both the decomposition and reconstruction processes; in particular, a tight compactly supported wavelet frame based on a multiresolution analysis employs finite masks during both processes. Nevertheless, until very recently, tight wavelet frames were hardly considered an issue in wavelet theory; the main reason for this was the lack of a general method for the construction of useful tight wavelet frames.

The paper [RS1] marks a significant change in this state-of-the-art: it laid a foundation for the theory of wavelet frames in general, thereby for tight wavelet frames, via the new notion of quasi-wavelet frames. In particular, the paper suggests a new "unitary extension principle" that allows one, at least in theory, to derive tight wavelet frames from any multiresolution analysis, regardless of any conditions of orthonormality or linear independence of the scaling functions. Furthermore, in [RS2] this unitary extension principle is employed to derive various compactly supported spline tight wavelet frames in higher dimensions, i.e., tight wavelet frames based on smooth piecewise-polynomials.

We show in this note that the theory of [RS1] and the construction methods of [RS2], when combined with the existing literature on self-affine tilings for general dilation matrices, lead to the following observation: given any spatial dimension $d$, any $d \times d$ dilation matrix $s$, and any smoothness parameter $k$, there exists a compactly supported tight wavelet system in $C^{k}\left(\mathbb{R}^{d}\right)$ which is $s$-invariant. In view of the present inability to construct, for the majority of dilation matrices, compactly supported smooth orthonormal wavelet bases, this result might be of practical interest. The details of this observation are the content of the next section.

\section{Smooth TIGHT WAVELET FRAMES}

We first review some of the very basic notions in multiresolution analysis, and then present results from [RS1], [RS2] that are pertinent to the discussion. We then recall some facts concerning self-affine tilings. Finally, all these facts are combined to yield the construction of the compactly supported smooth tight wavelet frames.

A dilation matrix $s$ on $\mathbb{R}^{d}$ is a $d \times d$ integer matrix whose spectrum lies outside the closed unit disc. It induces two closely related dilation operators on functions; on the "space domain" this is the operator

$$
D: f \mapsto|\operatorname{det} s|^{-1} f\left(s^{-1} \cdot\right),
$$

and on the frequency domain, the relevant operator is

$$
D_{*}: f \mapsto f\left(s^{*}\right)
$$

the two operators are connected by the formula $(D f)^{\wedge}=D_{*} \widehat{f}$. A function $\phi \in$ $L_{2}\left(\mathbb{R}^{d}\right)$ is said to be a scaling function (with respect to a given dilation matrix $s$ ), if in the Fourier domain it satisfies an equality of the following type:

$$
D_{*} \widehat{\phi}=\tau_{\phi} \widehat{\phi},
$$

where $\tau_{\phi}$ is some $2 \pi$-periodic function, often referred to as the mask function. The refinement equation (2.1) is equivalent to having $D \phi$ lie in the closed span $V_{0}$ of the integer translates of $\phi,[\mathrm{BDR}]$. 
Now, let $\Psi$ be any finite subset of $V_{0}$. The assumption $\psi \in V_{0}$ is equivalent to the existence of a $2 \pi$-periodic function, $\tau_{\psi}$, that satisfies the wavelet equation

$$
D_{*} \widehat{\psi}=\tau_{\psi} \widehat{\phi} .
$$

Let $\Gamma$ be the quotient group $\mathbb{Z}^{d} /\left(s \mathbb{Z}^{d}\right)$, and let $\widetilde{\Gamma}$ be its dual group, i.e., the quotient group $2 \pi\left(s^{*-1} \mathbb{Z}^{d} / \mathbb{Z}^{d}\right)$ (both of order $\left.|\operatorname{det} s|\right)$. We may always identify any set of representers of $\Gamma$ or $\widetilde{\Gamma}$ with the group itself. We then consider the following matrixvalued function $\Delta$ on $\mathbb{R}^{d}$, whose rows are indexed by $F:=\Psi \cup\{\phi\}$, whose columns are indexed by $\widetilde{\Gamma}$, and whose $(f, \gamma) \in F \times \widetilde{\Gamma}$ entry is defined as

$$
\Delta_{f, \gamma}(\omega)=E^{\gamma} \tau_{f}(\omega)=\tau_{f}(\omega+\gamma),
$$

where $E^{t}, t \in \mathbb{R}^{d}$, is the translation operator

$$
E^{t}: f \mapsto f(\cdot+t) .
$$

The unitary extension principle of [RS1] then reads as follows:

Result 2.2 ([RS1, Corollary 6.7]). In the above notations, if $\widehat{\phi}(0)=1$, and if the columns of matrix $\Delta(\omega)$ are orthonormal for almost all $\omega \in \mathbb{R}^{d}$, then the system defined by

$$
X:=\left\{D^{k} E^{j} \psi: \psi \in \Psi, j \in \mathbb{Z}^{d}, k \in \mathbb{Z}\right\}
$$

is a tight (wavelet) frame.

Remark. The original proof in [RS1] requires a very mild smoothness assumption (which does not even imply continuity); but it follows from a result in [CSS] that Result 2.2 is valid without any smoothness condition. In any event, since we aim at very smooth scaling functions $\phi$, we need not worry about this technical detail.

In principle, one may be able to construct a tight wavelet frame from any scaling function $\phi$ with mask $\tau_{0}$ by solving the following matrix extension problem: given the mask function $\tau_{0}$, find a (possibly large) number of periodic functions $\tau_{i}, i=$ $1, \ldots, r$, such that the column vectors $u_{\gamma}(\omega)=\left(\tau_{0}(\gamma+\omega), \ldots, \tau_{r}(\gamma+\omega)\right)^{T}, \gamma \in \widetilde{\Gamma}$, are orthonormal for almost all $\omega$. While that seems still to be a hard problem, a partial solution was provided by an inductive method in [RS2]. The objective of this note is to show that the class of refinable functions to which the [RS2]-method can be applied is broad enough to include compactly supported $\phi$ 's of arbitrarily high smoothness. But, first, we need to provide the necessary details of the [RS2]construction.

For this, let $\phi_{1}, \phi_{2}$ be refinable compactly supported distributions with respect to the same dilation matrix $s$, viz.,

$$
D_{*} \widehat{\phi}_{j}=\tau_{j} \widehat{\phi}_{j}, \quad j=1,2,
$$

with $\tau_{j}, j=1,2$, being $2 \pi$-periodic. Roughly speaking, the goal is to derive a wavelet system from the scaling function $\phi_{1} * \phi_{2}$, based on the ability to do this separately with each of the scaling functions $\phi_{j}, j=1,2$. Suppose, indeed, that we are able to use the unitary extension principle with respect to $\phi_{1}$, and to obtain a collection $\mathrm{T}_{1}=(\tau)_{\tau \in \mathrm{T}_{1}}$ of $2 \pi$-periodic functions that satisfy the extension principle - in other words, the columns of the matrix $\Delta(\omega)$ associated with $\tau_{1} \cup \mathrm{T}_{1}$ 
are orthonormal for almost all $\omega \in \mathbb{R}^{d}$. As with respect to $\phi_{2}$, the assumption here is even milder, viz., that we only need to find a collection $\mathrm{T}_{2}=(\tau)_{\tau \in \mathrm{T}_{2}}$ so that

$$
\left|\tau_{2}\right|^{2}+\sum_{\tau \in \mathrm{T}_{2}}|\tau|^{2}=1
$$

The method in [RS2] then provides a unitary extension to $\tau_{0}:=\tau_{1} D_{*} \tau_{2}$, which is the mask of the scaling function $\phi:=\phi_{1} * D \phi_{2}$ (instead of the expected extension to the mask $\tau_{1} \tau_{2}$ of $\phi_{1} * \phi_{2}$ ). The wavelet masks that correspond to $\phi$ are

$$
\mathrm{T}:=\mathrm{T}_{1} \cup\left(\tau_{1} D_{*} \mathrm{~T}_{2}\right) ;
$$

i.e., the masks of $T_{1}$ are augmented by the modified masks of $T_{2}$, where the modification is done by dilating each of these masks and then multiplying each by $\tau_{1}$. It is shown in $[\mathrm{RS} 2, \S 4]$, that $\tau_{0} \cup \mathrm{T}$ satisfies the unitary extension principle, and hence by Result 2.2 the wavelet system generated by $\Psi:=\left(\psi_{\tau}\right)_{\tau \in \mathrm{T}}$, where

$$
D_{*} \widehat{\psi}_{\tau}:=\tau \widehat{\phi},
$$

is a tight wavelet frame, provided that $\phi$ is in $L_{2}\left(\mathbb{R}^{d}\right)$, and not merely a distribution.

In order to construct smooth tight wavelet frames with respect to $s$, we first find tight frames that are not smooth at all, something that is relatively easy; we then use the outlined method to improve on the smoothness. For that, we first invoke the following simple lemma:

Lemma 2.3. For a given dilation matrix $s$ with determinant $n=|\operatorname{det} s|$, fix a set $\Gamma=\left\{\gamma_{1}, \ldots, \gamma_{n}\right\}$ of representers for the group $\mathbb{Z}^{d} / s \mathbb{Z}^{d}$, and a set $\widetilde{\Gamma}=\left\{g_{1}, \ldots, g_{n}\right\}$ of representers for the dual group. For any unitary $n \times n$ matrix $U:=\left(u_{j k}\right)$, set

$$
\tau_{j}(\omega)=n^{-1 / 2} \sum_{k=1}^{n} u_{j k} e^{i \gamma_{k} \cdot \omega} .
$$

Then the $n \times n$ matrix $\Delta$ with entries $E^{g_{k}} \tau_{j}, j, k=1, \ldots, n$, is unitary for all $\omega$.

Proof. Let $R(\omega)$ be the $n \times n$ matrix whose $(j, k)$-entry is $n^{-1 / 2} e^{i \gamma_{j} \cdot\left(\omega+g_{k}\right)}$. This matrix is unitary due to the well-known orthogonality relations of group characters (cf. for instance $[\mathrm{GH}]$, Lemma 5.1). This implies that $\Delta(\omega)$ is unitary, too, as the product $U R(\omega)$ of two unitary matrices.

The lemma, when combined with Result 2.2, can be used for the construction of tight wavelet frame in the following special case. Suppose $\Gamma$ is a set of representers for the group $\mathbb{Z}^{d} /\left(s \mathbb{Z}^{d}\right)$, and suppose that $\phi$ is a scaling function whose refinement mask $\tau_{\phi}$ has the special form

$$
\tau_{\phi}(\omega)=\sum_{\gamma \in \Gamma} a_{\gamma} e^{i \gamma \cdot \omega}
$$

so that (with $n:=|\operatorname{det} s|) a:=n^{1 / 2}\left(a_{\gamma}\right)_{\gamma \in \Gamma}$ is a unit vector. Then, there exists a unitary matrix $U$ whose first row is $a$, and the lemma, then, when combined with Result 2.2 provides us with a tight compactly supported wavelet system generated by $n-1$ functions. The next two results assert now that: (a) there exist $L_{2}$-scaling functions with mask of the form (2.4), and (b) these functions, though not really smooth, have a minimal amount of smoothness. 
The first result is due to [GM]:

Result 2.5 ([GM, Theorem 2]). Let $s$ be a dilation matrix, and $\Gamma$ a representer set of $\mathbb{Z}^{d} /\left(s \mathbb{Z}^{d}\right)$. Set, with $n:=|\operatorname{det} s|$,

$$
\tau:=n^{-1} \sum_{\gamma \in \Gamma} e^{i \gamma \cdot \omega}
$$

Then the dilation equation $D_{*} \widehat{\phi}=\tau \widehat{\phi}$ has an $L_{2}$-compactly supported solution, with non-zero mean value. Furthermore, up to a multiplicative constant, $\phi$ is the support function of a uniquely determined (compact) set $\Omega$ which satisfies $s \Omega=\Gamma+\Omega$.

The wavelets that can be constructed by combining Result 2.2 , Result 2.5 , and Lemma 2.3, generate a tight frame, but lack smoothness: each wavelet is piecewise constant. On the positive side, they inherit their smoothness properties from the corresponding scaling function, and that scaling function is known to be "minimally smooth".

Result 2.6 ([S, Lemma 3.1]). For any s-self-affine tile $\Omega$ (i.e., a non-null measurable set $\Omega$ satisfying $s \Omega=\Gamma+\Omega$ for some set of representers $\Gamma$ of $\left.\mathbb{Z}^{d} / s \mathbb{Z}^{d}\right)$, there exists $\epsilon>0$ such that

$$
|\widehat{\chi}(\omega)|=O\left(|\omega|^{-\epsilon}\right) .
$$

Here, $\chi$ is the support function of $\Omega$.

The statement in $[\mathrm{S}]$ assumes that the smallest $s$-invariant lattice containing $\Gamma$ equals $\mathbb{Z}^{d}$, but can be modified to yield our stronger statement. Since this strengthening is critical for the development here, we outline below the modified proof.

Proof of Result 2.6. With $\Omega$ and $\Gamma$ as in the result, one immediately obtains that

$$
D_{*} \widehat{\chi}=\tau \hat{\chi}
$$

with $\tau(\omega)=|\operatorname{det} s|^{-1} \sum_{\gamma \in \Gamma} e^{i \gamma \cdot \omega}$. Let $\Lambda$ be the smallest $s$-invariant lattice that contains $\Gamma$. Since $\Lambda$, as any lattice in $\mathbb{R}^{d}$, is finitely generated, it is easy to see that, for a sufficiently large $N$, the set

$$
\Gamma_{N}:=\sum_{j=0}^{N-1} s^{j} \Gamma
$$

generates $\Lambda$. Since we still have $s^{N} \Omega=\Gamma_{N}+\Omega$, it follows that $\chi$ is refinable also with respect to $s^{N}$, and with mask $|\operatorname{det} s|^{-N} \sum_{\gamma \in \Gamma_{N}} e^{i \gamma \cdot \omega}$. This allows one to assume (by replacing $\Gamma$ by $\Gamma_{N}$ if necessary) that the original $\Gamma$ generates $\Lambda$.

Let $\Lambda^{\perp}$ be the lattice dual to $\Lambda$ :

$$
\Lambda^{\perp}:=\left\{\omega \in \mathbb{R}^{d}: \lambda \cdot \omega \in 2 \pi \mathbb{Z} \text {, all } \lambda \in \Lambda\right\}=\left\{\omega \in \mathbb{R}^{d}: \gamma \cdot \omega \in 2 \pi \mathbb{Z} \text {, all } \gamma \in \Gamma\right\},
$$

with the second equality following from the fact that $\Gamma$ generates $\Lambda$. Then,

$$
|\tau(\omega)|=1 \Longleftrightarrow\left(e^{i \gamma \cdot \omega}=1 \text {, all } \gamma \in \Gamma\right) \Longleftrightarrow \omega \in \Lambda^{\perp} .
$$

Also, if $\omega \notin \Lambda^{\perp}$, but $s^{*} \omega \in \Lambda^{\perp}$, then $\omega$ is a non-trivial character of the (well-defined) group $\Lambda /(s \Lambda)$, and hence

$$
\tau(\omega)=\sum_{\gamma \in \Gamma} e^{i \gamma \cdot \omega}=0, \quad s^{*} \omega \in \Lambda^{\perp} \backslash\left(s^{*} \Lambda^{\perp}\right)
$$

(say, because every non-trivial character is orthogonal to the trivial one). 
The proof now proceeds in the same manner as in $[S]$, with the lattice $2 \pi \mathbb{Z}^{d}$ there being replaced by $\Lambda^{\perp}$. The essence of the proof is the observation that maximal chains of the form $\prod_{j=1}^{k} D_{*}^{j} \widehat{\chi}(\omega)$, where each $\left|D_{*}^{j} \widehat{\chi}(\omega)\right|$ is "near 1", must be preceded by a "near 0 " term $\widehat{\chi}(\omega)$. That observation requires only (2.7) and (2.8) above.

We can now easily combine Result 2.6 with the inductive method of [RS2]: we define

$$
\phi_{k}:=\phi * \underbrace{D \phi * D \phi * \ldots * D \phi}_{k \text { times }},
$$

with $\phi$ being the scaling function of Result 2.5. Then

$$
\left|\widehat{\phi}_{k}(\omega)\right|=O\left(|\omega|^{-k \epsilon}\right),
$$

and for $k$ large enough, $\phi_{k}$ has any desired smoothness.

The function $\phi_{k}$ is refinable with mask

$$
\tau^{(k)}:=\tau_{1}\left(D_{*} \tau_{1}\right)^{k},
$$

and the wavelets derived from $\phi_{k}$ are defined inductively as follows: for $k=0$, we use the construction that is detailed after Lemma 2.3. Next, we assume that $\mathrm{T}_{k-1}$ are the wavelet masks used in the derivation of a tight wavelet frame from $\phi_{k-1}$. Since we can write

$$
\phi_{k}=\phi_{k-1} * D \phi
$$

and since the refinement mask of $\phi_{k-1}$ is $\tau^{(k-1)}=\tau_{1}\left(D_{*} \tau_{1}\right)^{k-1}$, the [RS2]-method provides us with the new wavelets masks

$$
\mathrm{T}_{k}:=\mathrm{T}_{k-1} \cup\left(\tau^{(k-1)} D_{*} \mathrm{~T}_{0}\right),
$$

and the wavelets are then defined in the Fourier domain as

$$
D_{*} \widehat{\psi}_{\tau}:=\tau \widehat{\phi}_{k}, \quad \tau \in \mathrm{T}_{k} .
$$

Obviously, they have the same smoothness as $\phi_{k}$. Note that each inductive step increases the number of mother wavelets by $|\operatorname{det} s|-1$; hence $(k+1)(|\operatorname{det} s|-1)$ mother wavelets are derived from $\phi_{k}$.

We have thus proved:

Theorem 2.10. Given a dilation matrix $s$ on $\mathbb{R}^{d}$ and a smoothness parameter $n$, there exists a collection $\Psi$ of wavelet functions with compact support in $C^{n}\left(\mathbb{R}^{d}\right)$, such that

$$
X:=\left\{D^{k} E^{j} \psi: \psi \in \Psi, j \in \mathbb{Z}^{d}, k \in \mathbb{Z}\right\}
$$

is a tight frame.

Remark. In practice, one would like the smoothness of the sequence $\left(\phi_{k}\right)_{k}$ to increase as fast as possible. Using $k$-fold convolution of $D \phi$ is certainly far from being an optimal strategy for a speedy increase in the smoothness of $\phi_{k}$, as $k \rightarrow \infty$. An obvious modification is to take at each inductive step a different tile constructed as in Result 2.5 (by changing the set of representers $\Gamma$ that is used there). The construction details remain essentially unchanged: one only needs to modify the set $\mathrm{T}_{0}$ in (2.9) according to the nature of the fractal $\phi$ used. We refer to [DDL] for a related construction. 
Remark. A significant improvement in the above construction is available in case the matrix $s$ satisfies a relation

$$
s^{m}=(\operatorname{det} s)^{m} I
$$

for some integer $m$. In that event, one can smooth the scaling function $\phi$ of Result 2.5 by convolving it with a smooth box spline $\phi_{\xi}$ instead of $D \phi$. For a given vector $\xi \in \mathbb{Z}^{d} \backslash 0$ the box spline $\phi_{\xi}$ is defined as

$$
\widehat{\phi}_{\xi}(\omega):=\prod_{j=1}^{m} \int_{0}^{1} e^{-i\left(s^{j} \xi \cdot \omega\right) t} d t .
$$

The details of that construction are essentially in [RS2, §4], where the resulting wavelets were dubbed boxlets for the case of dyadic dilation. In that case each additional convolution increases the number of resulting wavelets by 1 only, and the smoothness of $\phi_{k}$ can be increased by as much as $m$ degrees per inductive step.

\section{REFERENCES}

[BDR] C. de Boor, R. DeVore and A. Ron, The structure of shift invariant spaces and applications to approximation theory, J. Functional Anal. 119 (1994), 37-78. Ftp site: anonymous@ftp.cs.wisc.edu/Approx file name several.ps MR 95g:46050

[CD] A. Cohen and I. Daubechies, Non-separable bidimensional wavelets bases, Rev. Math. Iberoamericana, Vol 9 (1993), 51-137. MR 94k:42047

[CSS] C.K. Chui, X.L. Shi and J. Stöckler, Affine frames, quasi-affine frames and their duals, CAT Report 372, Texas A\&M University, College Station, TX, 77843, June 1996.

[DDL] S. Dahlke, W. Dahmen, V. Latour, Smooth refinable functions and wavelets obtained from convolution products. Appl. Comp. Harm. Anal. 2 (1995), 68 - 84. MR 95m:42043

[D1] I. Daubechies, Orthonormal bases of compactly supported wavelets, Comm. Pure and Applied Math., 41 (1988), 909-996. MR 90m:42039

[D2] I. Daubechies, Ten lectures on wavelets, CBMS-NSF conference series in applied mathematics, Vol 61, SIAM, Philadelphia, 1992. MR 93e: 42045

[GH] K. Gröchenig and A. Haas, Self-similar lattice tilings. J. Fourier Anal. Appl. 1 (1994), 131-170. MR 96j:52037

[GM] K. Gröchenig and W. Madych, Multiresolution analysis, Haar bases, and self-similar tilings. IEEE Trans. Inform. Th. 38(2), (1992), 556-568. MR 93i:42001

[RS1] A. Ron and Z. Shen, Affine systems in $L_{2}\left(\mathbb{R}^{d}\right)$, the analysis of the analysis operator, J. Functional Anal., to appear. Ftp site: anonymous@ftp.cs.wisc.edu/Approx file affine.ps

[RS2] A. Ron and Z. Shen, Compactly supported tight affine spline frames in $L_{2}\left(\mathbb{R}^{d}\right)$, Math. Comp., to appear. Ftp site: anonymous@ftp.cs.wisc.edu/Approx file tight.ps

[S] R. S. Strichartz, Wavelets and self-affine tilings. Constr. Approx. 9 (1993), 327 - 346. MR 94f: 42039

Department of Mathematics U-9, University of Connecticut, Storrs, Connecticut 06269-3009

E-mail address: groch@math.uconn.edu

Department of Computer Science, University of Wisconsin-Madison, Madison, WisCONSIN 53706

E-mail address: amos@cs.wisc.edu 